\title{
Cosmological Perturbations from a Group Theoretical Point of View
}

\author{
István Szapudi ${ }^{1}$, Viktor G. Czinner ${ }^{2,3}$ \\ ${ }^{1}$ Institute for Astronomy, University of Hawaii, 2680 Woodlawn Dr., Honolulu, HI \\ 96815, USA \\ ${ }^{2}$ Department of Mathematics and Applied Mathematics, University of Cape Town, \\ Rondebosch, 7701, South Africa \\ ${ }^{3}$ Department of Theoretical Physics, KFKI Research Institute for Particle and \\ Nuclear Physics, H-1525 Budapest 114, P.O. Box 49, Hungary \\ E-mail: szapudi@ifa.hawaii.edu, czinner@rmki.kfki.hu
}

\begin{abstract}
We present a new approach to cosmological perturbations based on the theory of Lie groups and their representations. After re-deriving the standard covariant formalism from $S O(3)$ considerations, we provide a new expansion of the perturbed Friedmann-Lemaitre-Robertson-Walker (FLRW) metric in terms of irreducible representations of the Lorentz group. The resulting decomposition splits into (scalar, scalar), (scalar, vector) and (vector, vector) terms. These equations directly correspond to the standard Lifshitz classification of cosmological perturbations using scalar, vector and tensor modes which arise from the irreducible $S O(3)$ representation of the spatial part of the metric. While the Lorentz group basis matches the underlying local symmetries of the FLRW spacetime better than the $S O(3)$, the new equations do not provide further simplification compared to the standard cosmological perturbation theory. We conjecture that this is due to the fact that the $s o(3,1) \sim s u(2) \times s u(2)$ Lorentz algebra has no pair of commuting generators commuting with any of the translation group generators.
\end{abstract}

PACS numbers: 98.80.Jk, 02.20.Qs, 04.20.-q 


\section{Introduction}

Linear cosmological perturbation theory plays a fundamental role in our understanding of the evolution of small inhomogeneities of the universe scaling from quantum fluctuations to large scale structure formation. In an early paper [1], Lifshitz and Khalatnikov laid the groundwork for all subsequent research on the subject. The cosmological perturbations were expressed in spatially flat gauge, concentrating all fluctuations into the spatial part of the metric. The perturbations were then classified according to their transformation properties in the background spacetime as scalar, vector and tensor modes, and it was shown that the different modes do not mix in the linear theory. This is the celebrated "decomposition theorem".

Based on the above results, almost twenty years later, Bardeen introduced a gauge invariant formalism [2] which has since become the cornerstone of linear cosmological perturbation theory. The formalism has been further developed and generalized in subsequent works (see e.g. [3, 4]).

In the gauge dependent approach of Lifshitz and Khalatnikov [1], all gauge freedom is used to constrain the perturbations into the spatial domain. Due to the isotropy of the background FLRW spacetime, the spatial part of the perturbed metric is an $S O(3)$ tensor, and as such, it can be expanded into irreducible representations, an $l=0$ scalar (the trace of the spatial part of the metric) and an $l=2$ tensor (a traceless symmetric tensor). The latter has five independent components, corresponding to the $m=-2,-1,0,1,2$ eigenvalues of the rotation generator around an arbitrarily chosen direction. The classification of [1] corresponds to $|m|$ of these representations, where $|m|=0,1,2$ belongs to the scalar-, vector- and tensor modes respectively (note the slightly different and confusing terminology from group theory). In other words,

perturbations are classified according to how a mode responds to rotations around a chosen axis.

Interestingly in [2], Bardeen kept the same classification scheme even for gauge invariant perturbations. Once the whole metric is considered, it is locally transforming as an $S O(3,1)$, i.e. a Lorentz tensor. The Lorentz algebra describes transformations of the time-time and time-space components of the metric under infinitesimal so(3) rotations of space, a subalgebra of the full Lorentz algebra. It is easy to show that the time-time component of the metric transforms as a scalar with respect to spatial rotations, and the time-space part as a vector. As shown by Bardeen, the decomposition theorem still holds using the classification of the perturbations according to the $m$ eigenvalue (magnetic quantum number in physicist's terminology).

The above considerations provide a motivation to investigate the mathematical theory of cosmological perturbations from a group theoretical point of view. In addition, in the literature it is customary to start from the result that the spatial dependence of linear perturbations in the Fourier-space can be completely represented by the solutions of the generalized Helmholtz equation. The mathematical background of this result is that the symmetry group of the solutions of the Helmholtz equation, the Euclidean group 
(see e.g. [5]), is precisely the group of geometrical symmetries of the spatial part of the (homogeneous and isotropic) FLRW metric. Thus the expansion of the spatial part of the perturbations into solutions of the Helmholtz equation is essentially a harmonic analysis on the Euclidean group as a Lie group. This observation provides further motivation to examine closely the group theoretical structure behind perturbation theory.

To the best of our knowledge, the theory of cosmological perturbations, in general, has not been considered to date from the point of view of Lie groups, and the principal goal of the present paper is to develop an approach to cosmological perturbations based on the theory of Lie groups and their representations. More specifically, we investigate the theory using irreducible representations derived from the more relevant Lorentz algebra, and not just its $s o(3)$ subalgebra as usual. This is motivated by the observation that the spatially flat FLRW spacetime (which we only consider in this paper), i.e. the zeroth order cosmological solution, is conformally flat, and by the locally Lorentzian nature of the metric tensor. Our hope is to elucidate the role that symmetries play in the decomposition of the 10 Einstein equations into independent subsets based on the decomposition theorem.

The plan of the paper is as follows. In section 2, we present a quick overview of the standard covariant formalism; for further details please refer to [2, 3, 4] or, for a more informal introduction, [6]. In section [3, first we reproduce the standard covariant formalism from $S O(3)$ considerations, and then we expand the perturbations using irreducible representations of the full Lorentz algebra. In section 4, we present the perturbed field equations obtained from the Lorentz decomposition, and in section 5, we discuss gauge invariant quantities. Finally, in section 6 , we summarize our results and draw conclusions.

\section{Standard covariant formalism in a nutshell}

The background spacetime is described by the FLRW metric which we introduce in the conformal form

$$
d s^{2}=g_{\mu \nu} d x^{\mu} d x^{\nu}=a^{2}(\eta)\left(-d \eta^{2}+\gamma_{i j} d x^{i} d x^{j}\right),
$$

where $\eta$ is the conformal time variable and $\gamma_{i j}$ is the metric tensor for a 3-space of uniform spatial curvature $K$. Here, and throughout the paper, Greek indices run from 0 to 3 while Latin indices run from 1 to 3 .

As mentioned earlier, perturbations in various quantities are classified according to how they transform under spatial coordinate transformations in the background spacetime as scalar-, vector- and tensor modes. The homogeneity and isotropy of the background metric allows the separation of the time dependence and the spatial dependence, with the spatial dependence related to solutions of a generalized Helmholtzequation. Scalar-, vector- and tensor harmonics are solutions of the scalar-

$$
\nabla^{2} Q^{(0)}+k^{2} Q^{(0)}=0
$$


vector-

$$
\nabla^{2} Q_{i}^{(1)}+k^{2} Q_{i}^{(1)}=0
$$

and tensor

$$
\nabla^{2} Q_{i j}^{(2)}+k^{2} Q_{i j}^{(2)}=0
$$

Helmholtz-equations respectively, where $\nabla$ denotes the covariant derivative with respect to the spatial metric $\gamma_{i j}$.

In a spatially flat $(K=0)$ universe, the harmonics are essentially plane waves:

$$
\begin{aligned}
& Q^{(0)}=\exp (i \mathbf{k} \cdot \mathbf{x}), \\
& Q_{i}^{( \pm 1)}=\frac{-i}{\sqrt{2}}\left(\mathbf{e}_{1} \pm i \mathbf{e}_{2}\right)_{i} \exp (i \mathbf{k} \cdot \mathbf{x}), \\
& Q_{i j}^{( \pm 2)}=-\sqrt{\frac{3}{8}}\left(\mathbf{e}_{1} \pm i \mathbf{e}_{2}\right)_{i}\left(\mathbf{e}_{1} \pm i \mathbf{e}_{2}\right)_{j} \exp (i \mathbf{k} \cdot \mathbf{x}),
\end{aligned}
$$

where $\mathbf{x}=\left(x_{1}, x_{2}, x_{3}\right)$ and $\mathbf{e}_{1}, \mathbf{e}_{2}$ are unit vectors spanning the plane transverse to the wave vector $\mathbf{k} \equiv\left(k_{1}, k_{2}, k_{3}\right)$. The vector modes represent divergence-free (vorticity) vectors while the tensor modes are transverse and traceless, and represent gravitational waves:

$$
\nabla^{i} Q_{i}^{( \pm 1)}=0, \quad \nabla^{i} Q_{i j}^{( \pm 2)}=0, \quad \gamma^{i j} Q_{i j}^{( \pm 2)}=0 .
$$

The curl free vectors and the longitudinal components of tensors can be obtained from covariant derivatives of the scalar- and vector modes by

$$
\begin{aligned}
Q_{i}^{(0)} & =-k^{-1} \nabla_{i} Q^{(0)} \\
Q_{i j}^{(0)} & =\left(k^{-2} \nabla_{i} \nabla_{j}+\frac{1}{3} \gamma_{i j}\right) Q^{(0)}, \\
Q_{i j}^{( \pm 1)} & =-\frac{1}{2 k}\left[\nabla_{i} Q_{j}^{( \pm 1)}+\nabla_{j} Q_{i}^{( \pm 1)}\right],
\end{aligned}
$$

where $k=|\mathbf{k}|$.

A completely general perturbation of the gravitational field can be written as a linear combination of perturbations associated with individual spatial harmonics defined above, and no coupling between the different modes. Thus, a general perturbation to the FLRW metric can be represented for example as [2]

$$
\begin{aligned}
& g_{00}=-a^{2}(1+2 A), \\
& g_{0 i}=-a^{2} B_{i}, \\
& g_{i j}=a^{2}\left(\gamma_{i j}+2 H_{L} \gamma_{i j}+2 H_{T i j}\right),
\end{aligned}
$$

with

$$
\begin{aligned}
& A \equiv A\left(x^{k}, \eta\right) \text { - scalar potential; } \\
& B_{i} \equiv B_{i}\left(x^{k}, \eta\right) \text { - vector shift; } \\
& H_{L} \equiv H_{L}\left(x^{k}, \eta\right) \text { - scalar perturbation to the spatial curvature; } \\
& H_{T i j} \equiv H_{T i j}\left(x^{k}, \eta\right) \text { - trace free distortion to the spatial metric. }
\end{aligned}
$$


For the $k$ th harmonics, the scalar-, vector- and tensor components of the perturbed metric become

$$
\begin{aligned}
& A(\eta, \mathbf{x})=A(\eta, k) Q^{(0)}(\mathbf{x}), \\
& H_{L}(\eta, \mathbf{x})=H_{L}(\eta, k) Q^{(0)}(\mathbf{x}), \\
& B_{i}(\eta, \mathbf{x})=\sum_{m=-1}^{1} B^{(m)}(\eta, k) Q_{i}^{(m)}(\mathbf{x}), \\
& H_{T i j}(\eta, \mathbf{x})=\sum_{m=-2}^{2} H_{T}^{(m)}(\eta, k) Q_{i j}^{(m)}(\mathbf{x}) .
\end{aligned}
$$

Let us now rewrite the perturbed metric (6) in the form

$$
g_{\alpha \beta}=\stackrel{\circ}{g}_{\alpha \beta}+\delta g_{\alpha \beta} .
$$

It can be shown by simple calculations that according to the presented expansion, the first order part of the metric

$$
\begin{aligned}
\delta g_{00} & =-2 a^{2} A, \\
\delta g_{0 i} & =-a^{2} B_{i}, \\
\delta g_{i j} & =2 a^{2}\left(H_{L} \gamma_{i j}+H_{T i j}\right),
\end{aligned}
$$

with the choice of $\mathbf{k}=(0,0, k)$, can be decomposed as

$$
\delta g_{\alpha \beta}=\left(Q^{(0)} \sum_{j=1}^{10} o_{j} O_{j}\right)_{\alpha \beta},
$$

where the $o_{j}$ amplitudes are

$$
\begin{array}{rlrl}
o_{1} & =2 a^{2} A(\eta, k), & o_{2} & =2 a^{2} H_{L}(\eta, k), \\
o_{3} & =\frac{a^{2}}{\sqrt{2}} B^{(-1)}(\eta, k), & o_{4} & =a^{2} B^{(0)}(\eta, k), \quad o_{5}=\frac{a^{2}}{\sqrt{2}} B^{(+1)}(\eta, k), \\
o_{6} & =\sqrt{\frac{3}{2}} a^{2} H_{T}^{(-2)}(\eta, k), o_{7} & =\frac{a^{2}}{\sqrt{2}} H_{T}^{(-1)}(\eta, k), o_{8}=\frac{2 a^{2}}{3} H_{T}^{(0)}(\eta, k), \\
o_{9} & =\frac{a^{2}}{\sqrt{2}} H_{T}^{(+1)}(\eta, k), & o_{10} & =\sqrt{\frac{3}{2}} a^{2} H_{T}^{(+2)}(\eta, k),
\end{array}
$$

and $O_{j}$ are the following $10,4 \times 4$, symmetric matrices:

$$
\begin{array}{rlrl}
O_{1}=\left(\begin{array}{cccc}
-1 & 0 & 0 & 0 \\
0 & 0 & 0 & 0 \\
0 & 0 & 0 & 0 \\
0 & 0 & 0 & 0
\end{array}\right) ; & O_{2}=\left(\begin{array}{cccc}
0 & 0 & 0 & 0 \\
0 & 1 & 0 & 0 \\
0 & 0 & 1 & 0 \\
0 & 0 & 0 & 1
\end{array}\right) ; \\
O_{3}=\left(\begin{array}{cccc}
0 & i & 1 & 0 \\
i & 0 & 0 & 0 \\
1 & 0 & 0 & 0 \\
0 & 0 & 0 & 0
\end{array}\right) ; & O_{4}=\left(\begin{array}{cccc}
0 & 0 & 0 & i \\
0 & 0 & 0 & 0 \\
0 & 0 & 0 & 0 \\
i & 0 & 0 & 0
\end{array}\right) ; & O_{5}=\left(\begin{array}{cccc}
0 & i & -1 & 0 \\
i & 0 & 0 & 0 \\
-1 & 0 & 0 & 0 \\
0 & 0 & 0 & 0
\end{array}\right) ;
\end{array}
$$




$$
\begin{aligned}
& O_{6}=\left(\begin{array}{cccc}
0 & 0 & 0 & 0 \\
0 & -1 & i & 0 \\
0 & i & 1 & 0 \\
0 & 0 & 0 & 0
\end{array}\right), O_{7}=\left(\begin{array}{cccc}
0 & 0 & 0 & 0 \\
0 & 0 & 0 & -1 \\
0 & 0 & 0 & i \\
0 & -1 & i & 0
\end{array}\right) ; \quad O_{8}=\left(\begin{array}{cccc}
0 & 0 & 0 & 0 \\
0 & 1 & 0 & 0 \\
0 & 0 & 1 & 0 \\
0 & 0 & 0 & -2
\end{array}\right) \\
& O_{9}=\left(\begin{array}{cccc}
0 & 0 & 0 & 0 \\
0 & 0 & 0 & -1 \\
0 & 0 & 0 & -i \\
0 & -1 & -i & 0
\end{array}\right) ; \quad O_{10}=\left(\begin{array}{cccc}
0 & 0 & 0 & 0 \\
0 & -1 & -i & 0 \\
0 & -i & 1 & 0 \\
0 & 0 & 0 & 0
\end{array}\right)
\end{aligned}
$$

This decomposition provides a unique expansion of the metric perturbations up to constant normalization factors.

The parametrization of the matter stress-energy tensor of a perfect fluid in the standard formalism is given by

$$
\begin{aligned}
& T_{0}^{0}=-\rho-\delta \rho, \\
& T_{i}^{0}=(\rho+p)\left(v_{i}-B_{i}\right), \\
& T_{0}^{i}=-(\rho+p) v_{i}, \\
& T_{j}^{i}=(p+\delta p) \delta^{i}{ }_{j}+p \Pi^{i}{ }_{j},
\end{aligned}
$$

where $\delta \rho$ is a scalar density perturbation, $v_{i}$ is a vector velocity perturbation to the spacelike part of the zeroth order four velocity $u_{\alpha}=[a(\eta), 0,0,0]$ of the fluid, and $\Pi_{i j}$ is a tensor anisotropic stress perturbation.

In the present paper we prefer to work with lower indices, thus for the perturbation of the stress-energy tensor we use

$$
\delta T_{\alpha \beta}=\stackrel{\circ}{g}_{\alpha \mu} \delta T_{\beta}^{\mu}-\stackrel{\circ}{g}_{\alpha \nu} \delta g^{\nu \tau} \stackrel{\circ}{\tau \beta} .
$$

By taking into account the explicit form of $\stackrel{\circ}{T}_{\alpha \beta}$ as

$$
\stackrel{\circ}{T}_{\alpha \beta}=(\rho+p) u_{\alpha} u_{\beta}+p \stackrel{\circ}{g}_{\alpha \beta},
$$

with the given parametrization of the metric and the matter perturbations we get

$$
\begin{aligned}
& \delta T_{00}=a^{2}(\delta \rho+2 \rho A), \\
& \delta T_{0 i}=-a^{2}\left[(\rho+p)\left(v_{i}-B_{i}\right)+p B_{i}\right], \\
& \delta T_{i j}=a^{2}\left[\delta p \delta_{i j}+p\left[\Pi_{i j}+2\left(H_{L} \gamma_{i j}+H_{T i j}\right)\right]\right] .
\end{aligned}
$$

Now, in a completely analog fashion to the metric perturbations (given in (10)), we can decompose the perturbations of the stress-energy tensor as

$$
\delta T_{\alpha \beta}=\left(Q^{(0)} \sum_{j=1}^{10} T_{j} O_{j}\right)_{\alpha \beta},
$$

where, for the kth harmonics, the scalar-, vector- and tensor components of the perturbations become

$$
\begin{aligned}
& \delta \rho(\eta, \mathbf{x})=\delta \rho(\eta, k) Q^{(0)}(\mathbf{x}), \\
& \delta p(\eta, \mathbf{x})=\delta p(\eta, k) Q^{(0)}(\mathbf{x}),
\end{aligned}
$$


Cosmological Perturbations from a Group Theoretical Point of View

$$
\begin{aligned}
& v_{i}(\eta, \mathbf{x})=\sum_{m=-1}^{1} v^{(m)}(\eta, k) Q_{i}^{(m)}(\mathbf{x}) \\
& \Pi_{i j}(\eta, \mathbf{x})=\sum_{m=-2}^{2} \Pi^{(m)}(\eta, k) Q_{i j}^{(m)}(\mathbf{x})
\end{aligned}
$$

and the $T_{j}$ coefficients are

$$
\begin{aligned}
T_{1} & =-a^{2}(\delta \rho+2 \rho A), & T_{2} & =a^{2}\left(\delta p+2 p H_{L}\right), \\
T_{3} & =\frac{a^{2}}{\sqrt{2}}\left[(\rho+p) v^{(-1)}-\rho B^{(-1)}\right], & T_{4} & =\frac{a^{2}}{\sqrt{2}}\left[(\rho+p) v^{(0)}-\rho B^{(0)}\right], \\
T_{5} & =\frac{a^{2}}{\sqrt{2}}\left[(\rho+p) v^{(+1)}-\rho B^{(+1)}\right], & T_{6} & =\sqrt{\frac{3}{8}} a^{2} p\left(\Pi^{(-2)}+2 H_{T}^{(-2)}\right), \\
T_{7} & =\frac{a^{2} p}{2 \sqrt{2}}\left(\Pi^{(-1)}+2 H_{T}^{(-1)}\right), & T_{8} & =\frac{a^{2} p}{3}\left(\Pi^{(0)}+2 H_{T}^{(0)}\right), \\
T_{9} & =\frac{a^{2} p}{2 \sqrt{2}}\left(\Pi^{(+1)}+2 H_{T}^{(+1)}\right), & T_{10} & =\sqrt{\frac{3}{8}} a^{2} p\left(\Pi^{(+2)}+2 H_{T}^{(+2)}\right) .
\end{aligned}
$$

With this parametrization it is straightforward, although tedious, to derive the Einstein's field equations at linear order. The explicit forms of the scalar-, vector- and tensor equations are provided for example in the equations (17), (20) and (22) in [6] respectively. We will use them as a reference hereafter, and also present them here in the spatially flat $(K=0)$ case for our latter convenience.

Scalar equations

$$
\begin{aligned}
& k^{2}\left[H_{L}+\frac{1}{3} H_{T}+\frac{\dot{a}}{a}\left(\frac{B}{k}-\frac{\dot{H}_{T}}{k^{2}}\right)\right]=4 \pi G a^{2}\left[\delta \rho+3 \frac{\dot{a}}{a}(\rho+p) \frac{v-B}{k}\right], \\
& k^{2}\left(A+H_{L}+\frac{1}{3} H_{T}\right)+\left(\frac{d}{d \eta}+2 \frac{\dot{a}}{a}\right)\left(k B-\dot{H}_{T}\right)=-8 \pi G a^{2} p \Pi, \\
& \frac{\dot{a}}{a} A-\dot{H}_{L}-\frac{1}{3} \dot{H}_{T}=4 \pi G a^{2}(\rho+p) \frac{v-B}{k}, \\
& {\left[2 \frac{\ddot{a}}{a}-2\left(\frac{\dot{a}}{a}\right)^{2}+\frac{\dot{a}}{a} \frac{d}{d \eta}-\frac{k^{2}}{3}\right] A-\left[\frac{d}{d \eta}+\frac{\dot{a}}{a}\right]\left(\dot{H}_{L}+\frac{k B}{3}\right)=4 \pi G a^{2}\left(\delta p+\frac{1}{3} \delta \rho\right) .}
\end{aligned}
$$

Vector equations

$$
\begin{gathered}
k B^{( \pm 1)}-\dot{H}_{T}^{( \pm 1)}=16 \pi G a^{2}(\rho+p) \frac{v^{( \pm 1)}-B^{( \pm 1)}}{k} \\
{\left[\frac{d}{d \eta}+2 \frac{\dot{a}}{a}\right]\left(k B^{( \pm 1)}-\dot{H}_{T}^{( \pm 1)}\right)=-8 \pi G a^{2} p \Pi^{( \pm 1)}}
\end{gathered}
$$

Tensor equations

$$
\left[\frac{d^{2}}{d \eta^{2}}+2 \frac{\dot{a}}{a} \frac{d}{d \eta}+k^{2}\right] H_{T}^{( \pm 2)}=8 \pi G a^{2} p \Pi^{( \pm 2)} .
$$




\section{Perturbation theory using irreducible representations}

In the present section, we re-derive the perturbed field equations of the standard formalism with more explicit reference to group theory. First we consider the expansion of the metric perturbations according to irreducible representation of the $S O(3)$ group, and show that the corresponding equations are identical with the ones presented in the previous section. After this preparation, we consider the expansion according to an irreducible representation of $S O(3,1)$, the full Lorentz group. We explicitly compute a (trace-orthogonal) basis for the decomposition of a second grade $S O(3,1)$ tensor according to the $s u(2) \times s u(2)$ decomposition of the Lorentz algebra. We present the corresponding linear field equations in the next section.

\subsection{The $S O(3)$ case}

To specify our notation, the Lorentz algebra is defined by the following commutation relations

$$
\left[L_{\mu \nu}, L_{\rho \sigma}\right]=i g_{\nu \rho} L_{\mu \sigma}-i g_{\mu \rho} L_{\nu \sigma}-i g_{\nu \sigma} L_{\mu \rho}+i g_{\mu \sigma} L_{\nu \rho} .
$$

Note that we are using "physicist" convention, i.e. Hermitian generators, instead of the anti-Hermitian (or "mathematician") convention. The spatial part of the algebra can be cast in a familiar form

$$
\left[J_{j}, J_{k}\right]=i \epsilon_{j k l} J_{l},
$$

where $J_{j}=\frac{1}{2} \epsilon_{j k l} L_{k l}$ is the Hodge dual of the spatial generators. This subalgebra is equivalent to that of $s u(2)$ or $s o(3)$. With the usual boost generators $K_{i}=L_{0 i}$ we have the following relations

$$
\left[K_{j}, K_{k}\right]=-i \epsilon_{j k l} K_{l}, \quad\left[J_{j}, K_{k}\right]=i \epsilon_{j k l} K_{l} .
$$

For the FLRW solution in a spatially flat universe, the metric tensor is conformally flat and its spatial part is conformally Euclidean. Moreover, the spatial part of the metric is a locally $S O(3)$ tensor. Therefore it makes sense to expand the spatial part of the metric in terms of irreducible representations under $S O(3)$. The irreducible representations of a $3 \times 3$, symmetric matrix are the trace $(l=0$, scalar $)$, and a rank- 2 , traceless, symmetric tensor $(l=2$ mode). Here $l$ corresponds to the eigenvalues of the Casimir operator (total angular momentum in physics terminology), and for $l=2$ there are five modes which correspond to the five degrees of freedom in a rank-2, traceless, symmetric matrix. These are usually parametrized according to the eigenvalue of the rotation generator in the direction of an arbitrarily chosen z-axis. The corresponding eigenvalues (magnetic quantum numbers in physics) are $m=-2,-1,0,1,2$. These $V_{l, m}$ tensors are traditionally called "polarization operators", and we quote their explicit forms from [7] as given below. 


$$
\begin{aligned}
& V_{2,2}=\frac{1}{2}\left(\begin{array}{ccc}
-1 & -i & 0 \\
-i & 1 & 0 \\
0 & 0 & 0
\end{array}\right), \quad V_{2,1}=\frac{1}{2}\left(\begin{array}{ccc}
0 & 0 & 1 \\
0 & 0 & i \\
1 & i & 0
\end{array}\right), \quad V_{2,0}=\frac{1}{\sqrt{6}}\left(\begin{array}{ccc}
1 & 0 & 0 \\
0 & 1 & 0 \\
0 & 0 & -2
\end{array}\right), \\
& V_{2,-1}=\frac{1}{2}\left(\begin{array}{ccc}
0 & 0 & -1 \\
0 & 0 & i \\
-1 & i & 0
\end{array}\right), \quad V_{2,-2}=\frac{1}{2}\left(\begin{array}{ccc}
-1 & i & 0 \\
i & 1 & 0 \\
0 & 0 & 0
\end{array}\right), \quad V_{0,0}=\frac{1}{\sqrt{3}}\left(\begin{array}{ccc}
1 & 0 & 0 \\
0 & 1 & 0 \\
0 & 0 & 1
\end{array}\right) .
\end{aligned}
$$

These matrices yield a natural basis on which to expand $3 \times 3$ symmetric matrices, such as the spatial part of the metric tensor. Careful comparison reveals that the previously introduced $\mathrm{O}_{2}$ matrix corresponds to $V_{0,0}$, i.e. the trace is an $S O(3)$ scalar, while $O_{6} \ldots O_{10}$ directly correspond to $V_{2,2} \ldots V_{2,-2}$, the $S O(3)$ tensor modes with the five possible values of $m$; the three dimensional representations were extended into four dimensions with rows and columns of 0's.

The time-time part of the metric tensor $g_{00}$ does not respond to $S O(3)$ rotations, i.e. it is an $S O(3)$ scalar. In four dimensions it is represented with the matrix $O_{1}$. It can be shown (c.f. eq. (21)) that under rotations $g_{0 i}$ is an $S O(3)$ vector. The spherical basis for a vector is $g_{03}, g_{01} \pm i g_{02}$. This is represented with the matrices $O_{4}, O_{5}$ and $O_{3}$ respectively (up to a $-i$ factor introduced for convenience sake). These latter matrices contain zeros for their spatial part.

As it was noted in the introduction, and it also follows from the above argument, the standard parametrization of the metric (based on the solution of the Helmholtz equations) directly corresponds to an irreducible representation according to $S O(3)$, i.e. spatial rotations. The resulting 10 parameters repackage the 10 degrees of freedom in a symmetric $4 \times 4$ matrix according to irreducible representations of $S O(3)$.

These matrices are suitable parameters for an expansion motivated by the $S O(3)$ invariance of the spatial part of the FLRW solution at fixed time, and the local $S O(3)$ invariance of space. The homogeneity (translation invariance) of the universe can be further utilized with Fourier transformation, i.e. an expansion according to the irreducible representation of the translation generators with eigenvalue $k$. In particular, once a corresponding $k$-mode is fixed for the transform, we can choose the orientation of our $z$-axis (global rotation invariance). This will zero out derivatives in the other two directions. Conventionally this idea is expressed using "transverse" and "longitudinal" modes in real space.

We thus recovered the perturbation theory, described in section 2, by expanding the perturbed metric according to the above representation of the $S O(3)$ group, and Fourier transforming after choosing the $z$-axis such that it is parallel with the $k$-mode in question. This procedure exploited the maximal symmetry group of the Helmholtz equation, the Euclidean group, that is also the group of geometrical symmetries of the spatial part of the background solution. The corresponding perturbed field equations are identical to the ones of the standard covariant formalism. Using computer algebra, 
we explicitly recovered the equations of [6] based on the matrix formulation presented above.

\subsection{The $S O(3,1)$ case}

Let us now take into consideration the larger local symmetry of the metric tensor, the $S O(3,1)$ group, for constructing a perturbation theory according to irreducible representations. This is also motivated by the conformal flatness of the spatially flat FLRW solution. The calculation is exactly analogous to the exposition of the $S O(3)$ perturbation theory above. A popular parametrization of the $s o(3,1)$ algebra splits into two commuting $s u(2)$ algebras. Then for each $s u(2)$ we can use the familiar theory of irreducible representations.

To find the irreducible representations, we used a spinor formalism: in each $s u(2)$ 's the irreducible representations are found by the standard way of symmetrizing spinor expressions. The direct product of the two commuting irreducible representations form a representation of $s o(3,1)$. For constructing spinor representations we used Infeld-van der Waerden symbols (see e.g. [8])

$$
\begin{aligned}
& \sigma_{A B^{\prime}}^{0}=\frac{1}{\sqrt{2}}\left(\begin{array}{ll}
1 & 0 \\
0 & 1
\end{array}\right), \quad \sigma_{A B^{\prime}}^{1}=\frac{1}{\sqrt{2}}\left(\begin{array}{ll}
0 & 1 \\
1 & 0
\end{array}\right), \\
& \sigma_{A B^{\prime}}^{2}=\frac{1}{\sqrt{2}}\left(\begin{array}{cc}
0 & i \\
-i & 0
\end{array}\right), \quad \sigma_{A B^{\prime}}^{3}=\frac{1}{\sqrt{2}}\left(\begin{array}{cc}
1 & 0 \\
0 & -1
\end{array}\right)
\end{aligned}
$$

to map $g_{\mu \nu}$ into $g_{A A^{\prime} B B^{\prime}}$, where primed and unprimed indices live in the two commuting $s u(2)$ 's. The symmetric $4 \times 4$ metric tensor is equivalent to a direct product of two vector representations for each of the $s u(2)$ 's. One can find matrices analogous to $O_{j}$ by symmetrizing in the primed and unprimed indices. After explicit symmetrization we have one scalar mode: the $4 D$ trace $(S$ in (24) $)$. The traceless $4 \times 4$ tensor is a direct product of two vector modes, each of which can be classified according to the respective $m$ eigenvalue, corresponding to the generator according to an arbitrarily chosen $z$-axis in each representation. The pair $\left(m, m^{\prime}\right)$, both take three possible values, $-1,0,1$, giving 9 possibilities. These $1+9$ modes cover the 10 degrees of freedom in $4 \times 4$ symmetric tensors. We present the corresponding $S$ and $S_{m, m^{\prime}}$ matrices explicitly:

$$
\begin{array}{rlrl}
S_{1,-1}= & \left(\begin{array}{cccc}
1 & 0 & 0 & 1 \\
0 & 0 & 0 & 0 \\
0 & 0 & 0 & 0 \\
1 & 0 & 0 & 1
\end{array}\right) ; & S_{1,0}=\left(\begin{array}{cccc}
0 & 1 & i & 0 \\
1 & 0 & 0 & 1 \\
i & 0 & 0 & i \\
0 & 1 & i & 0
\end{array}\right) ; \\
S_{1,1}=\left(\begin{array}{cccc}
0 & 0 & 0 & 0 \\
0 & 1 & i & 0 \\
0 & i & -1 & 0 \\
0 & 0 & 0 & 0
\end{array}\right) ; & S_{0,-1}=\left(\begin{array}{cccc}
0 & 1 & -i & 0 \\
1 & 0 & 0 & 1 \\
-i & 0 & 0 & -i \\
0 & 1 & -i & 0
\end{array}\right) ;
\end{array}
$$


Cosmological Perturbations from a Group Theoretical Point of View

$$
\begin{aligned}
& S_{0,0}=\left(\begin{array}{cccc}
1 & 0 & 0 & 0 \\
0 & 1 & 0 & 0 \\
0 & 0 & 1 & 0 \\
0 & 0 & 0 & -1
\end{array}\right) ; \quad S_{0,1}=\left(\begin{array}{cccc}
0 & 1 & i & 0 \\
1 & 0 & 0 & -1 \\
i & 0 & 0 & -i \\
0 & -1 & -i & 0
\end{array}\right) \\
& S_{-1,-1}=\left(\begin{array}{cccc}
0 & 0 & 0 & 0 \\
0 & 1 & -i & 0 \\
0 & -i & -1 & 0 \\
0 & 0 & 0 & 0
\end{array}\right) ; \quad S_{-1,0}=\left(\begin{array}{cccc}
0 & 1 & -i & 0 \\
1 & 0 & 0 & -1 \\
-i & 0 & 0 & i \\
0 & -1 & i & 0
\end{array}\right) \text {; } \\
& S_{-1,1}=\left(\begin{array}{cccc}
1 & 0 & 0 & -1 \\
0 & 0 & 0 & 0 \\
0 & 0 & 0 & 0 \\
-1 & 0 & 0 & 1
\end{array}\right) ; \quad S=\left(\begin{array}{cccc}
-1 & 0 & 0 & 0 \\
0 & 1 & 0 & 0 \\
0 & 0 & 1 & 0 \\
0 & 0 & 0 & 1
\end{array}\right) \text {. }
\end{aligned}
$$

Similar to the $S O(3)$ case, we can decompose the perturbed metric according to the above $s u(2) \times s u(2)$ representation as

$$
\delta g_{\alpha \beta}=a^{2}\left[s S+\sum_{m, m^{\prime}=-1}^{1} s_{m, m^{\prime}} S_{m, m^{\prime}}\right]_{\alpha \beta},
$$

where

$$
s_{m, m^{\prime}}=s_{m, m^{\prime}}\left(x^{\alpha}\right), \quad s=s\left(x^{\alpha}\right),
$$

and the stress-energy tensor perturbation as

$$
\begin{aligned}
& \delta T_{00}=-a^{2}\left[(t+\rho s) S+\sum_{m, m^{\prime}=-1}^{1}\left(t_{m, m^{\prime}}-\rho s_{m, m^{\prime}}\right) S_{m, m^{\prime}}\right]_{00}, \\
& \delta T_{0 i}=a^{2}\left[\begin{array}{c}
(-t+p s) S+\sum_{m, m^{\prime}=-1}^{1}\left(-t_{m, m^{\prime}}+p s_{m, m^{\prime}}\right) S_{m, m^{\prime}} \\
]_{0 i}
\end{array}\right. \\
& \delta T_{i j}=a^{2}\left[\begin{array}{c}
(t+\rho s) S+\sum_{m, m^{\prime}=-1}^{1}\left(t_{m, m^{\prime}}+p s_{m, m^{\prime}}\right) S_{m, m^{\prime}} \\
]_{i j}
\end{array},\right.
\end{aligned}
$$

with

$$
t_{m, m^{\prime}}=t_{m, m^{\prime}}\left(x^{\alpha}\right), \quad t=t\left(x^{\alpha}\right) .
$$

Once we expand Einstein's equation into the parameters corresponding to the above matrices, fix a $k$-mode for Fourier transforming, and choose the $z$-axis parallel with this mode, we obtain the perturbation equations.

\section{Perturbation equations}

The decomposition of the perturbations according to the $S_{m, m^{\prime}}$ and $S$ matrices results three independent groups of equations. A simple relation maps our decomposition to that of the traditional one, namely $m+m^{\prime} \rightarrow m$, where $\left(m, m^{\prime}\right)$ corresponds to the $s u(2) \times s u(2)$, and $m$ corresponds to the traditional so(3) scalar-vector-tensor 
decomposition. For example $(0,1)$ or $(-1,0)$ correspond to vector modes, etc. In addition, our parameter $s$ also corresponds to a scalar mode in scalar-vector-tensor terms.

Accordingly, the first group consists of two independent wave equations for $s_{1,1}$ and $s_{-1,-1}$ as

$$
\begin{aligned}
& \ddot{s}_{1,1}+\frac{2 \dot{a}}{a} \dot{s}_{1,1}+k^{2} s_{1,1}=16 \pi G a^{2} t_{1,1}, \\
& \ddot{s}_{-1,-1}+\frac{2 \dot{a}}{a} \dot{s}_{-1,-1}+k^{2} s_{-1,-1}=16 \pi G a^{2} t_{-1,-1},
\end{aligned}
$$

where, and throughout the paper, an overdot denotes the derivative with respect to the conformal time $\eta$. The above equations are then clearly identical with the tensor equations of the standard formalism provided in Sec.2, and thus the $s_{1,1}$ and $s_{-1,-1}$ amplitudes correspond to gravitational waves while the $t_{1,1}$ and $t_{-1,-1}$ amplitudes correspond to anisotropic stress perturbations.

The second group of equations consists of two decoupled systems, one for $s_{1,0}$ and $s_{0,1}$, and another for $s_{0,-1}$ and $s_{-1,0}$. The two systems are identical.

$$
\begin{aligned}
& k\left(s_{0,1}+s_{1,0}\right)-i\left(\dot{s}_{0,1}-\dot{s}_{1,0}\right)=\frac{16 \pi G a^{2}}{k}\left[t_{0,1}+t_{1,0}-(\rho+p)\left(s_{0,1}+s_{1,0}\right)\right], \\
& k\left(s_{-1,0}+s_{0,-1}\right)-i\left(\dot{s}_{-1,0}-\dot{s}_{0,-1}\right)=\frac{16 \pi G a^{2}}{k}\left[t_{-1,0}+t_{0,-1}-(\rho+p)\left(s_{-1,0}+s_{0,-1}\right)\right], \\
& {\left[\frac{d}{d \eta}+\frac{2 \dot{a}}{a}\right]\left(i k\left(s_{0,1}+s_{1,0}\right)+\left(\dot{s}_{0,1}-\dot{s}_{1,0}\right)=16 \pi G a^{2}\left(t_{0,1}-t_{1,0}\right),\right.} \\
& {\left[\frac{d}{d \eta}+\frac{2 \dot{a}}{a}\right]\left(i k\left(s_{-1,0}+s_{0,-1}\right)+\left(\dot{s}_{-1,0}-\dot{s}_{0,-1}\right)=16 \pi G a^{2}\left(t_{-1,0}-t_{0,-1}\right) .\right.}
\end{aligned}
$$

Thus, the $s_{1,0}, s_{0,1}, t_{1,0}, t_{0,1}$ and $s_{-1,0}, s_{0,-1}, t_{-1,0}, t_{0,-1}$ degrees of freedom in the $s u(2) \times s u(2)$ representation correspond to the vector amplitudes of the $s o(3)$ representation.

The remaining group of equations is the following coupled system of the $s_{1,-1}, s_{0,0}$, $s_{-1,1}, s$ and $t_{1,-1}, t_{0,0}, t_{-1,1}, t$ amplitudes,

$$
\begin{aligned}
& \frac{3 \dot{a}^{2}}{a^{2}}\left(s_{0,0}+s_{1,-1}+s_{-1,1}-s\right)+\frac{\dot{a}}{a}\left[\dot{s}_{0,0}+\dot{s}_{1,-1}+\dot{s}_{-1,1}+3 \dot{s}-2 i k\left(s_{1,-1}-s_{-1,1}\right)\right] \\
& +k^{2}\left(s_{0,0}+s\right)=-8 \pi G a^{2}\left(t_{0,0}+t_{1,-1}+t_{-1,1}+t+2 \rho s\right), \\
& {\left[\frac{d}{d \eta}+\frac{2 \dot{a}}{a}\right]\left(\dot{s}_{1,-1}+\dot{s}_{-1,1}-2 \dot{s}_{0,0}+2 i k\left(s_{-1,1}-s_{1,-1}\right)\right)-k^{2}\left(s_{1,-1}+s_{-1,1}-2 s\right)} \\
& =16 \pi G a^{2}\left(t_{1,-1}+t_{-1,1}-2 t_{00}\right), \\
& i\left[\frac{\dot{a}}{a}\left(s-s_{1,-1}-s_{-1,1}-s_{0,0}\right)-\dot{s}_{0,0}-\dot{s}\right]=\frac{8 \pi G a^{2}}{k}\left(t_{1,-1}-t_{-1,1}-(\rho+p)\left(s_{1,-1}-s_{-1,1}\right)\right), \\
& {\left[6\left(\frac{\dot{a}^{2}}{a^{2}}-\frac{\ddot{a}}{a}\right)-\frac{d}{d \eta}+k^{2}\right]\left(s_{0,0}+s_{1,-1}+s_{-1,1}-s\right)-4\left[\frac{\dot{a}}{a}\left(\dot{s}_{0,0}+\dot{s}_{1,-1}+\dot{s}_{-1,1}\right)+\ddot{s}\right]+} \\
& 2 i k\left[\left(\frac{\dot{a}}{a}+\frac{d}{d \eta}\right)\left(s_{1,-1}-s_{-1,1}\right)\right]=16 \pi G a^{2}(t-\rho s) .
\end{aligned}
$$

This system is clearly equivalent to the scalar equations of the so(3) representation. 
In general, one can find the following one-to-one correspondence between the standard $s o(3)$ and the $s u(2) \times s u(2)$ parametrization of the perturbation amplitudes

$$
\begin{array}{ll}
s_{1,1}=-\sqrt{\frac{3}{2} H_{T}^{(+2)},} & t_{1,1}=-\sqrt{\frac{3}{8}} p \Pi^{(+2)}, \\
s_{-1,-1}=-\sqrt{\frac{3}{2} H_{T}^{(-2)},} & t_{-1,-1}=-\sqrt{\frac{3}{8}} p \Pi^{(-2)}, \\
s_{ \pm 1,0}=\frac{i B^{( \pm 1)} \mp H_{T}^{( \pm 1)}}{2 \sqrt{2}}, & t_{ \pm 1,0}=\frac{1}{2 \sqrt{2}}\left((\rho+p) i v^{( \pm 1)} \mp \frac{p \Pi^{( \pm 1)}}{2}\right), \\
s_{0, \pm 1}=\frac{i B^{( \pm 1)} \pm H_{T}^{( \pm 1)}}{2 \sqrt{2}}, & t_{ \pm 1,0}=\frac{1}{2 \sqrt{2}}\left((\rho+p) i v^{( \pm 1)} \pm \frac{p \Pi^{( \pm 1)}}{2}\right), \\
s_{1,-1}=\frac{H_{L}-A+i B^{(0)}}{2}-\frac{H_{T}^{(0)}}{3}, & t_{1,-1}=-\frac{1}{2}\left(\delta p+\delta \rho+\frac{p \Pi^{(0)}}{3}-(\rho+p) i v^{(0)}\right) \\
s_{-1,1}=\frac{H_{L}-A-i B^{(0)}}{2}-\frac{H_{T}^{(0)}}{3}, & t_{-1,1}=-\frac{1}{2}\left(\delta p+\delta \rho+\frac{p \Pi^{(0)}}{3}+(\rho+p) i v^{(0)}\right) \\
s_{0,0}=\frac{H_{L}-A}{2}+\frac{2}{3} H_{T}^{(0)}, & t_{0,0}=\frac{p \Pi^{(0)}}{3}-\frac{\delta p+\delta \rho}{2} \\
s=\frac{A+3 H_{L}}{2}, & t=\frac{3 \delta p+\delta \rho}{2} .
\end{array}
$$

Despite the property that the Lorentz group matches the underlying local symmetries of the FLRW spacetime better than $S O(3)$, no further simplifications arise in the field equations compared to the standard decomposition theorem. We could not further decouple the above system. The new equations based on the irreducible representation of the $S O(3,1)$ group through an $s u(2) \times s u(2)$ decomposition of the Lorentz algebra appear to be equivalent to the $S O(3)$ approach.

\section{Gauge invariant quantities}

One of the key features of Bardeen's formalism is the systematic determination of the gauge invariant variables. It is therefore important to show how these quantities can be obtained under the $s u(2) \times s u(2)$ representation. In the present section we derive the transformation properties of the metric perturbation amplitudes $s$ and $s_{m, m^{\prime}}$, and compute the corresponding gauge invariant metric quantities. For the matter perturbations the computations are completely analogous.

The most general gauge transformation is induced by the coordinate transformation

$$
\bar{x}^{\alpha}=x^{\alpha}+\xi^{\alpha}\left(x^{\beta}\right),
$$

where $\xi^{\alpha}$ is of the same order as $s$ and $s_{m, m^{\prime}}$.

The changes in the metric tensor can be computed from the transformation law

$$
\bar{g}_{\alpha \beta}\left(\bar{x}^{\gamma}\right)=\frac{\partial x^{\mu}}{\partial \bar{x}^{\alpha}} \frac{\partial x^{\nu}}{\partial \bar{x}^{\beta}} g_{\mu \nu}\left(x^{\kappa}\right),
$$


and the scale factors in $\bar{g}_{\alpha \beta}$ and $g_{\alpha \beta}$ at linear order are related by

$$
a(\bar{\eta})=a(\eta)\left[1+\frac{\dot{a}}{a} \xi^{0}\right]
$$

For the linear metric perturbations one can obtain the relation

$$
\delta \bar{g}_{\alpha \beta}\left(\bar{x}^{\gamma}\right)=\delta g_{\alpha \beta}\left(x^{\kappa}\right)-a^{2}(\eta)\left[\frac{2 \dot{a}}{a} \xi^{0} \eta_{\alpha \beta}+\eta_{\alpha \mu} \xi_{, \beta}^{\mu}+\eta_{\nu \beta} \xi_{, \alpha}^{\nu}\right],
$$

where $\eta_{\mu \nu}$ is the Minkowski metric tensor, and a comma denotes partial derivative. After inserting the explicit $s u(2) \times s u(2)$ decomposed forms of $\delta g_{\alpha \beta}$ and $\delta \bar{g}_{\alpha \beta}$ using (25) we get

$$
\left.\left[\begin{array}{c}
\bar{s} S+\sum_{m, m^{\prime}=-1}^{1} \bar{s}_{m, m^{\prime}} S_{m, m^{\prime}} \\
]_{\alpha \beta}
\end{array}\right]_{m, m^{\prime}=-1}^{1} s_{m, m^{\prime}} S_{m, m^{\prime}}\right]_{\alpha \beta}-\frac{2 \dot{a}}{a} \xi^{0} \eta_{\alpha \beta}-\eta_{\alpha \mu} \xi_{, \beta}^{\mu}-\eta_{\nu \beta} \xi_{, \alpha}^{\nu} .
$$

The final form of the change in the amplitudes is then given by

$$
\begin{aligned}
& \bar{s}_{1,1}=s_{1,1}, \\
& \bar{s}_{-1,-1}=s_{-1,-1}, \\
& \bar{s}_{1,0}=s_{1,0}+\frac{1}{4}\left(-\dot{\xi}^{1}+i \dot{\xi}^{2}-i k \xi^{1}-k \xi^{2}\right), \\
& \bar{s}_{-1,0}=s_{-1,0}+\frac{1}{4}\left(-\dot{\xi}^{1}-i \dot{\xi}^{2}+i k \xi^{1}-k \xi^{2}\right), \\
& \bar{s}_{0,1}=s_{0,1}+\frac{1}{4}\left(-\dot{\xi}^{1}+i \dot{\xi}^{2}+i k \xi^{1}+k \xi^{2}\right), \\
& \bar{s}_{0,-1}=s_{0,-1}+\frac{1}{4}\left(-\dot{\xi}^{1}-i \dot{\xi}^{2}-i k \xi^{1}+k \xi^{2}\right), \\
& \bar{s}_{1,-1}=s_{1,-1}+\frac{1}{2}\left(\dot{\xi}^{0}-\dot{\xi}^{3}+i k \xi^{0}-i k \xi^{3}\right), \\
& \bar{s}_{-1,1}=s_{-1,1}+\frac{1}{2}\left(\dot{\xi}^{0}+\dot{\xi}^{3}-i k \xi^{0}-i k \xi^{3}\right), \\
& \bar{s}_{0,0}=s_{0,0}+\frac{1}{2}\left(\dot{\xi}^{0}+i k \xi^{3}\right), \\
& \bar{s}=s+\frac{1}{2}\left(-\dot{\xi}^{0}-\frac{2 \dot{a}}{a} \xi^{0}-i k \xi^{3}\right) .
\end{aligned}
$$

The $s_{1,1}$ and $s_{-1,-1}$ amplitudes are gauge invariant, just as we expected since they correspond to the tensor modes in the standard formalism. Similarly to the field equations, the remaining amplitudes decouple into two groups. The amplitudes belonging to the vector modes in the standard formalism depend only on $\xi^{1}$ and $\xi^{2}$, while the amplitudes belonging to the scalar modes depend only on $\xi^{0}$ and $\xi^{3}$.

Consequently, from the first group, one can construct two gauge invariant variables

$$
\begin{aligned}
& \Psi^{(+1)}=i\left(s_{1,0}+s_{0,1}\right)+\frac{\dot{s}_{0,1}-\dot{s}_{1,0}}{k}, \\
& \Psi^{(-1)}=i\left(s_{-1,0}+s_{0,-1}\right)+\frac{\dot{s}_{-1,0}-\dot{s}_{0,-1}}{k},
\end{aligned}
$$

which coincide with Bardeen's gauge invariant vector amplitudes. 
From the second group one can again construct two gauge invariant quantities

$$
\begin{aligned}
\Phi_{1} & =s+s_{0,0}+\frac{\dot{a}}{a}\left[\frac{2 i}{k}\left(s_{-1,1}-s_{1,-1}\right)+\frac{1}{k^{2}}\left(\dot{s}_{-1,1}+\dot{s}_{1,-1}-2 \dot{s}_{0,0}\right)\right], \\
\Phi_{2} & =s-s_{0,0}-s_{1,-1}-s_{-1,1}+\frac{2 i}{k}\left[\dot{s}_{-1,1}-\dot{s}_{1,-1}+\frac{\dot{a}}{a}\left(s_{-1,1}-s_{1,-1}\right)\right] \\
& -\frac{1}{k^{2}}\left[2 \ddot{s}_{0,0}-\ddot{s}_{1,-1}-\ddot{s}_{-1,1}+\frac{\dot{a}}{a}\left(2 \dot{s}_{0,0}-\dot{s}_{1,-1}-\dot{s}_{-1,1}\right)\right]
\end{aligned}
$$

which, as one can check, are related to Bardeen's scalar variables as

$$
\Phi_{1} \equiv 2 \Phi_{H} \quad \text { and } \quad \Phi_{2} \equiv 2 \Phi_{A} .
$$

As it is well known from the standard so(3) theory (see e.g. [3]), any gauge invariant variable that can be constructed from $s_{1,0}, s_{-1,0}, s_{0,1}$ and $s_{0,-1}$ and their time derivatives can be written as a linear combination of $\Psi^{(+1)}$ and $\Psi^{(-1)}$ and their time derivatives with coefficients of arbitrary functions of time. The same is true for the amplitudes $s_{1,-1}$, $s_{-1,1}, s_{0,0}$ and $s$ with the variables $\Phi_{1}$ and $\Phi_{2}$. In addition, corresponding pair of gauge invariant variables can be obtained from the perturbations of the stress-energy tensor to form the complete, closed set of gauge invariant variables (up to linear transformations).

\section{Summary and Conclusions}

We have developed an approach to linear cosmological perturbation theory based on Lie groups and their representations. After a short overview on the standard formalism, based on the decomposition by the solutions of the generalized Helmholtz equation, we explicitly reproduced the theory from the irreducible representations of the $S O(3)$ group, and utilizing the translation symmetry by Fourier transforming. This procedure completely exploited the maximal symmetry group of the Helmholtz equation (i.e. the Euclidean group), the group of geometrical symmetries of the spatial part of the background FLRW solution.

As an application of our new approach, we expanded the perturbations according to irreducible representations of the Lorentz algebra through the popular $s u(2) \times s u(2)$ decomposition. This expansion was motivated by the fact that the spatially flat FLRW cosmological metric is conformally flat, and that the local symmetry group of the metric is the Lorentz group, of which $S O(3)$ is only a subgroup. Our hope was to elucidate the role that local symmetries play in the decomposition of the perturbed field equations, and check whether it is possible to obtain a simpler system of equations than that of the standard formalism.

As a result we presented a new decomposition by (scalar, scalar), (scalar, vector), and (vector, vector) terms. We showed that these can be mapped directly into the standard scalar-vector-tensor classification. Furthermore, we showed that the resulting equations are simply related to the equations of [6]. The decomposition according to the larger Lorentz group did not produce a finer split of the perturbed field equations than the standard one based on the subgroup $S O(3)$. Our calculation elucidated the explicit role of the local $S O(3)$ and $S O(3,1)$ invariance as well as translation invariance. 
In particular, in the $s o(3)$ theory, the $J_{3}$ generator commutes with the generator of translations in the $z$-direction, a property that is crucial for simplifying the equations. On the contrary, in the $s u(2) \times s u(2)$ representation, no $s o(3,1)$ generator commutes with any of the translation generators. We speculate that this is the reason why the larger group, which most naturally matches the local symmetries of the metric tensor and the conformal form of the zeroth order cosmological solution, did not provide a simpler system of equations than the standard formulation.

Our calculation provides a blue-print for decomposing tensor quantities using group theoretical considerations in different perturbation theories of Einstein's field equations. Possible generalizations of the group theoretical formulation for cosmological perturbation theory includes different representations of the Lorentz (or the conformal Poincaré) group, using for example the full conformal group generators for decomposition, fully spinorial perturbation theory, or generalization to higher dimensions using the corresponding local symmetries of the metric tensor. The presented group theoretical approach could also be useful beyond the linear order, or considering inhomogeneous cosmological models. Furthermore the formalism may be applied to perturbation theory of other, not necessarily cosmological, solutions of the Einstein equations with different background symmetries.

\section{Acknowledgments}

Many parts of the calculations were performed and checked using the computer algebra programs MATHEMATICA 7, MAPLE 13 and GRTensorII. I.S. acknowledges support from NASA grants NNG06GE71G and NNX10AD53G, and from the Polányi Program of the Hungarian National Office for Research and Technology (NKTH). V.G.Cz. was supported by the National Research Foundation of South Africa and the Hungarian National Research Fund, OTKA No. K67790 grant.

\section{References}

[1] Lifshitz E M and Khalatnikov I M 1963 Advances in Physics 12185

[2] Bardeen J M 1980 Phys. Rev. D 221882

[3] Kodama H and Sasaki M 1984 Prog. Theor. Phys. Suppl. 781

[4] Mukhanov V A, Feldman H A and Brandenberger R H 1992 Physics Reports 215203

[5] Miller W 1977 Symmetry and Separation of Variables (Addison-Wesley Publishing Company, Reading, Massachusetts)

[6] Hu W 2003 Covariant Linear Perturbation Formalism, Summer school lectures: 2002, Astroparticle Physics and Cosmology (Abdus Salam ICTP, Trieste) p 149 (Preprint astro-ph/0402060)

[7] Varshalovich D A, Moskalev A N and Khershonskii V K 1988 Quantum Theory of Angular Momentum (Singapore: World Scientific Pub. Co. Inc.) p 54

[8] O'Donell P 2003 Introduction to 2-Spinors in General Relativity (Singapore: World Scientific Publishing Co. Pte. Ltd.) p 24 